\title{
Resolution Quality and Atom Positions in Sub-Ångström Electron Microscopy
}

\author{
Michael A. O’Keefe*, Lawrence F. Allard** and Douglas A. Blom**
}

*Materials Sciences Division, LBNL 2-200, 1 Cyclotron Road, Berkeley, CA 94720, USA

**Metals \& Ceramics Division, Oak Ridge National Laboratory, Oak Ridge, TN 37831-6064, USA

John Cowley pioneered use of transmission electron microscopy (TEM) for high-resolution imaging and helped spur improvements in resolution that enabled researchers to pinpoint the positions of all but the lightest atoms within a crystal structure [1]. Sub-Ångström capabilities allow imaging of even the lightest atoms. Initially achieved with software aberration correction (focal-series reconstruction of the specimen exit-surface wave) [2], sub-Ångström imaging will become commonplace for next-generation electron microscopes with hardware-corrected lenses and monochromated electron beams [3]. Currently, advanced HR-TEMs can image columns of light atoms (carbon, oxygen, nitrogen) in complex structures, including the lithium atoms present in battery materials [4].

The ability to determine whether an image peak represents one single atom (or atom column) instead of several depends on the resolution of the HR-(S)TEM. Rayleigh's resolution criterion, an accepted standard in optics, was derived as a means for judging when two image intensity peaks from two sources of light (stars) are distinguishable from a single source [5]. Atom spacings closer than the Rayleigh limit have been resolved in HR-TEM [6], suggesting that it may be useful to consider other limits, such as the Sparrow resolution criterion [7]. From the viewpoint of the materials scientist, it is important to be able to use the image to determine whether an image feature represents one or more atoms (resolution), and where the atoms (or atom columns) are positioned relative to one another (resolution quality). When atoms and the corresponding image peaks are separated by more than the Rayleigh limit of the HR-(S)TEM, it is possible to adjust imaging parameters so that relative peak positions in the image correspond to relative atom positions in the specimen. When atoms are closer than the Rayleigh limit, we must find the relationship of the peak position to the atom position by peak fitting [8] or, if we have a suitable model, by image simulation [9].

Figure 1 shows a series of pairs of Bessel functions, $2 \mathrm{~J}_{1}(\mathrm{x}) / \mathrm{x}$, and their sums. At Rayleigh separation the sum has a central minimum $26.5 \%$ below the maxima. At closer spacing, the depth of the central minimum decreases until it just flattens out at the Sparrow separation [7]. Bessel function spacing is given as a Rayleigh-Sparrow parameter $Q_{R S}=\left(d_{X}-d_{S}\right) /\left(d_{R}-d_{S}\right)$, equal to 1 when the Bessel spacing $\mathrm{d}_{\mathrm{X}}$ corresponds to Rayleigh spacing $\mathrm{d}_{\mathrm{R}}$ and to 0 at Sparrow spacing $\mathrm{d}_{\mathrm{S}}$. Figure 1 illustrates how the separation parameter $\mathrm{P}_{\mathrm{TP}}$ of the "twin peaks" changes from 100\% of Bessel separation at Rayleigh resolution to 37\% at Sparrow resolution as $\mathrm{Q}_{\mathrm{RS}}$ drops from 1 to 0 . Over this range, the central dip decreases from $26.5 \%$ to zero and the twin-peak height increases by $11 \%$ (Fig. 2). Experimentally, a $\mathrm{Q}_{\mathrm{RS}}$ value can be obtained from the dip and the Bessel-Rayleigh spacing ratio calculated from $\left(\mathrm{d}_{\mathrm{X}} / \mathrm{d}_{\mathrm{R}}\right)=\mathrm{Q}_{\mathrm{RS}}+\left(1-\mathrm{Q}_{\mathrm{RS}}\right) \mathrm{d}_{\mathrm{S}} / \mathrm{d}_{\mathrm{R}}$. Given that $\mathrm{d}_{\mathrm{S}} / \mathrm{d}_{\mathrm{R}}$ is equal to 0.79 , then $\left(\mathrm{d}_{\mathrm{X}} / \mathrm{d}_{\mathrm{R}}\right)=0.21 \mathrm{Q}_{\mathrm{RS}}+0.79$. To find atom separation in an image with $\mathrm{Q}_{\mathrm{RS}}<1$, we measure the central dip and twin-peak values, find the corresponding $\mathrm{P}_{\mathrm{TP}}$ value (Fig. 3), and divide it into the measured twin-peak separation.

The insert in figure 3 shows an image of diamond in $<110>$ orientation obtained with the LBNL oneÅngström microscope (OAM). The image (3a) clearly shows the $0.89 \AA$ dumbbell twin peaks [6]. Although the $0.89 \AA$ spacing is resolved, profiles $(3 \mathrm{~b})$ show that resolution is closer to Sparrow than to Rayleigh. From the measured central dip of $7.4 \%$, the Rayleigh-Sparrow parameter is 0.43 (red dot in Fig. 2). Atom column spacing can be obtained from the twin-peak $\mathrm{P}_{\mathrm{TP}}$ value of $85 \%$ when $\mathrm{Q}_{\mathrm{RS}}$ is 0.43 (Fig. 2). This $\mathrm{P}_{\mathrm{TP}}$ value compares very well with the actual twin-peak separation that was measured from the known $0.89 \AA$ atom separation as $86 \%$ of atom separation (red dot in Fig. 3 ).

The Rayleigh-Sparrow parameter $\mathrm{Q}_{\mathrm{RS}}$ reveals the "resolution quality." $\mathrm{Q}_{\mathrm{RS}}$ values greater than 1 indicate a clearly resolved twin peak, while values between 1 and 0 mean a lower-quality resolution and an image with peaks displaced from the relative atom positions. The depth of the twin-peak minimum can be used to determine the value of $\mathrm{Q}_{\mathrm{RS}}$ and the true separation of the atom peaks that sum to produce the twin peak in the image. The Rayleigh-Sparrow parameter can be used to refine relative atom positions in defect images where atoms are closer than the Rayleigh limit of the HR(S)TEM, reducing the necessity for full image simulations from large defect models [10]. 
[1] J. M. Cowley \& S. Iijima Z. Naturforsch. 27a (1972) 445-451.

[2] M.A. O'Keefe et al. Ultramicroscopy 89, 4: (2001) 215-241.

[3] A.R. Lupini, A. Borisevich, W.H.Sides, Jr. \& S. J. Pennycook, Science 305 (2004) 5691: 1741-1741.

[4] Y. Shao-Horn, L. Croguennec, C. Delmas, E.C. Nelson \& M.A. O’Keefe, Nature Mat. 2 (2003) 464-467.

[5] Lord Rayleigh, Philosophical Magazine 8 (1876) 261-274.

[6] M.A. O'Keefe, E.C. Nelson, Y.C. Wang \& A. Thust, Philosophical Mag. B 81 (2001) 11, 1861-1878.

[7] C.M. Sparrow, Astrophys. Journal (1916) 44, 76-86.

[8] M.A. O'Keefe \& L.F. Allard, Microscopy \& Microanalysis 9, suppl.2: (2004) 1002-1003.

[9] M.A. O'Keefe, P.R. Buseck and S. Iijima, Nature 274 (1978) 322-324.

[10] Supported by Director, Office of Science, Office of Basic Energy Sciences, Materials Science Division, DOE (contract DE-AC03-76SF00098), and Asst. Sec. for EERE, Office of FreedomCAR and Vehicle Tech., HTML User Program, ORNL, managed by UT-Battelle, LLC for DOE (contract DE-AC05-00OR22725).

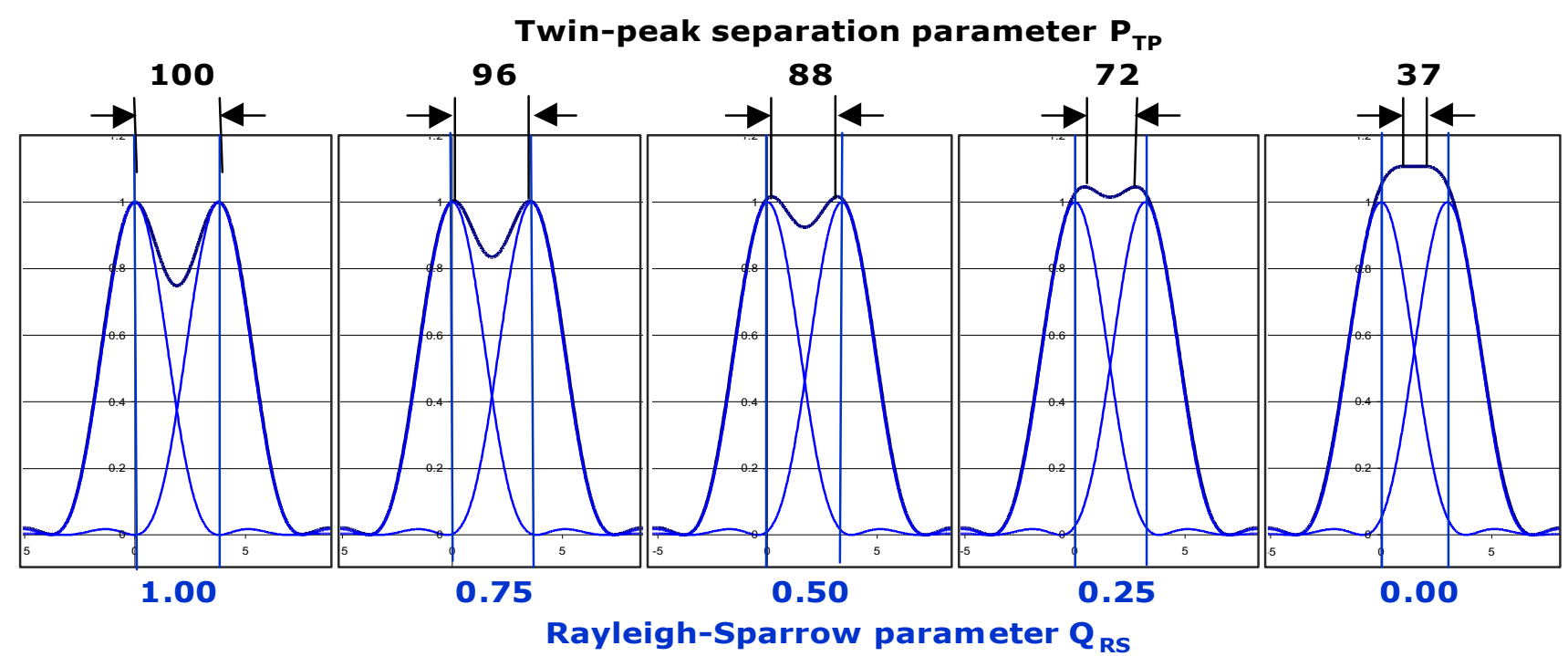

Fig. 1. Individual peaks of Bessel functions $2 \mathrm{~J}_{1}(\mathrm{x}) / \mathrm{x}$ plotted at separations ranging from Rayleigh (left) to Sparrow resolution (right) with the resultant summed "twin peaks." Arrows show the twinpeak separation parameter $\mathrm{P}_{\mathrm{TP}}$ as a percentage of the separation of the original Bessel peaks.

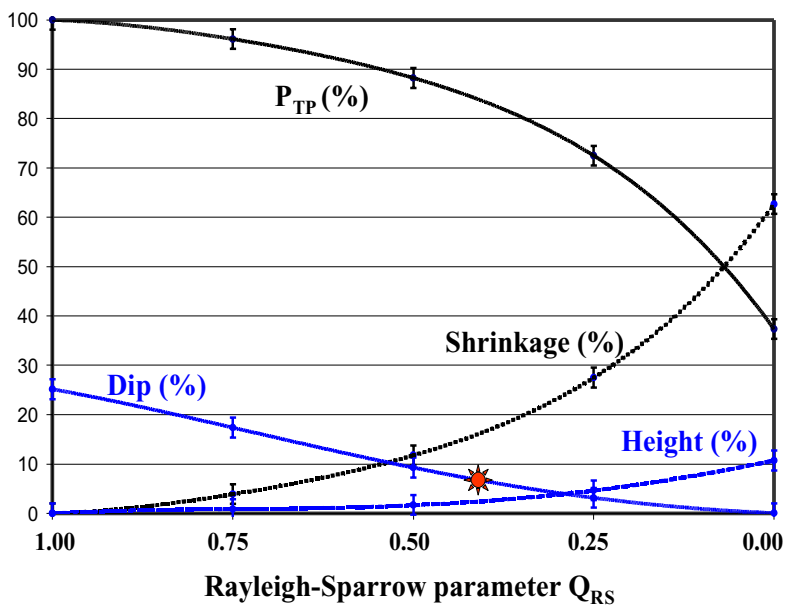

Fig. 2. From Rayleigh resolution (left) to Sparrow (right), the twin-peak separation parameter $\mathrm{P}_{\mathrm{TP}}$ falls (and shrinkage increases), the central dip decreases from $26.5 \%$ to zero, and the height increase goes from zero to $11 \%$.

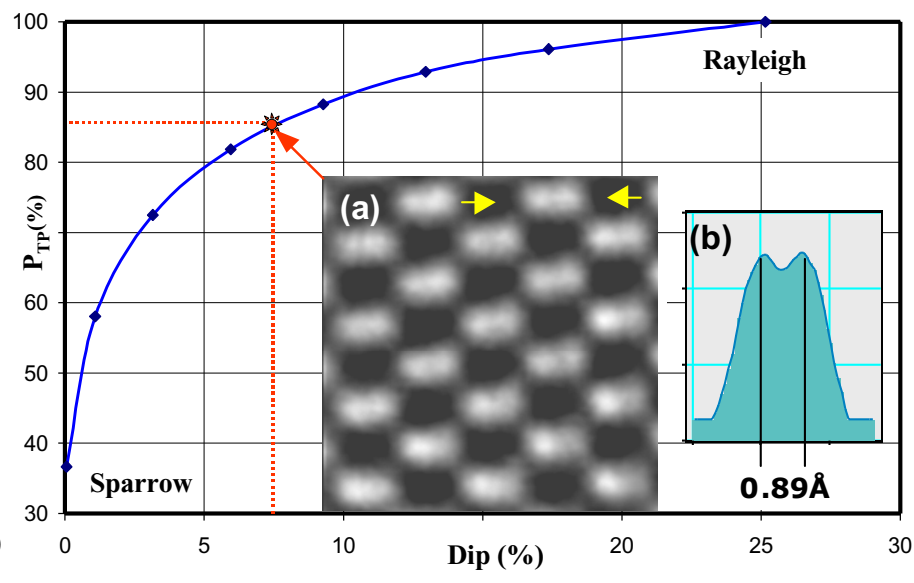

Fig. 3. Bessel plot data show how $\mathrm{P}_{\mathrm{TP}}$ varies with twin-peak dip (blue line). (a) Experimental OÅM image shows diamond dumbbells [6]. (b) Profile of diamond twin-peak shows dip of 7.4\%. Atom separation is known to be $0.89 \AA$ (marked), giving a twin-peak parameter $\mathrm{P}_{\mathrm{TP}}$ of $86 \%$ (red dot). 